\title{
Improved actions of the staggered fermion
}

\author{
Yubing Luo $^{\mathrm{a}}$ \\ ${ }^{a}$ Columbia University, Department of Physics, New York, NY 10027
}

We have studied $O\left(a^{2}\right)$ improved lattice QCD with the staggered fermion by using Symanzik's program. We find that there are 5 dimension- 6 fermion bilinears and gauge operators. In addition, there are 10 four-fermion operators which are absent at the tree-level and tadpole-improved tree-level.

\section{INTRODUCTION}

To remove $O\left(a^{2}\right)$ errors by using Symanzik's program, we must find all possible dimension- 6 terms which are scalars under the lattice symmetry transformations. Then, we add them to the original action and adjust their coefficients so that low-momentum physical quantities do not have $O\left(a^{2}\right)$ errors.

When he proposed this program, Symanzik applied it to scalar fields and proved the consistency of the improvement program to all orders of perturbation theory [1]. Lüscher and Weisz applied this program to pure gauge theory and developed the concept of on-shell improved action which demands only the improvement of all physical (on-shell) quantities [2]. Sheikholeslami and Wohlert applied this program to Wilson fermions and proposed a "Clover" term to remove all $O(a)$ errors from Wilson fermions [3]. Naik applied this program to Dirac-Kähler fermions and proposed a three-link term 四. Although the staggered fermion and the Dirac-Kähler fermion formalisms are the same in the free case, they are quite different when the gauge interaction is included. DiracKähler fermions have two drawbacks: First, the $U(1)_{o} \otimes U(1)_{e}$ symmetry does not prevent the gauge interaction from producing non-zero mass counterterms, therefore the bare mass parameters have to be tuned in the continuum limit, similar to Wilson fermions; Second, there is no massless Goldstone boson [5]. Therefore, in most applications, the standard staggered fermion formalism is used. Thus, it is important to investigate the improved action for the staggered fermion case.

Sharpe's pioneering work showed that there is no $O(a)$ term for the staggered fermion 6 . Thus the leading order terms in the improved action for staggered fermions is $O\left(a^{2}\right)$. In this talk, I will present all independent dimension- 6 terms, and show which terms remain after a spectrumconserving transformation of the fields. A detailed discussion of these results can be found in ref. [7].

\section{IMPROVED ACTION}

The improved action consists of two parts, the gauge action and fermion action:

$S=S_{G}+S_{F}$.

The improved gauge action contains 3 independent dimension- 6 operators and can be written as:

$S_{G}=\frac{2}{g_{0}^{2}}\left[c_{0} \mathcal{L}_{0}+c_{1} \mathcal{L}_{1}+c_{2} \mathcal{L}_{2}+c_{3} \mathcal{L}_{3}\right]$.

where $\mathcal{L}_{0}$ denotes the loops consists of 4 links, and $\mathcal{L}_{1}, \mathcal{L}_{2}$, and $\mathcal{L}_{3}$ are 3 sets of loops consisting of 6 links. The 4 coefficients satisfy a normalization condition:

$c_{0}+8 c_{1}+8 c_{2}+16 c_{3}=1$.

The improved fermion action can be written as:

$$
\begin{array}{r}
S_{F}=\bar{\chi}(\not D+m) \chi+a^{2} \sum_{i=1}^{7} b_{i} \mathcal{O}_{i} \\
+a^{2} \sum_{i=1}^{18} b_{i}^{\prime} \mathcal{F}_{i}+O\left(a^{3}\right) .
\end{array}
$$

It contains 7 fermion bilinears $\mathcal{O}_{i}$ in addition to the usual Dirac action and 18 four-fermion operators $\mathcal{F}_{i}$. 
Therefore, there are 25 possible dimension- 6 operators which can be added to the original lattice action.

\subsection{Fermion bilinears}

The 7 fermion bilinears are the linear combinations of the pattern: $\mathcal{D}^{3}, \mathcal{D}^{2} \not D, \not D D^{2}, \mathcal{D} \not D \mathcal{D}, \not D^{3}$, $m \not^{2}$ and $m \mathcal{D}^{2}$. They can be written as:

$$
\begin{aligned}
& \mathcal{O}_{1}=\bar{\chi} \mathcal{D}^{3} \chi, \\
& \mathcal{O}_{2}=\bar{\chi} \frac{1}{2}\left(\mathcal{D}^{2} \not D-\not D \mathcal{D}^{2}\right) \chi, \\
& \mathcal{O}_{3}=\bar{\chi} \frac{1}{2}\left(\mathcal{D}^{2} \not D+\not D \mathcal{D}^{2}-2 \not{D}^{3}\right) \chi, \\
& \mathcal{O}_{4}=\bar{\chi}\left(\mathcal{D}^{2} \not D+\not D \mathcal{D}^{2}-2 \mathcal{D} \not D \mathcal{D}\right) \chi \text {, } \\
& \mathcal{O}_{5}=\bar{\chi} \not{D}^{3} \chi . \\
& \mathcal{O}_{6}=m \bar{\chi} \not^{2} \chi, \\
& \mathcal{O}_{7}=m \bar{\chi} \mathcal{D}^{2} \chi \text {. }
\end{aligned}
$$

$\mathcal{O}_{1}$ is the Naik's term. $\mathcal{O}_{4}$ is equivalent to the MILC's "fat link" term [8]. $\mathcal{O}_{6}$ and $\mathcal{O}_{7}$ violate the $U_{A}(1)$ symmetry and therefore depend explicitly on the mass parameter.

\subsection{Four-fermion operators}

The 18 four-fermion operators can be divided into 4 groups. The first group contains one operator:

$\mathcal{F}_{1}=\sum_{x, a} \bar{\chi}(x) t^{a} \chi(x) \sum_{e} \bar{\chi}(x+e) t^{a} \chi(x+e)$,

where $t^{a}$ are the 8 generators of $S U(3)$ color group and the sum over $e$ is a sum over the 8 possible lattice displacements of length " 1 ". The second group contains one operator:

$\mathcal{F}_{2}=\sum_{x, a} \bar{\chi}(x) t^{a} \chi(x) \sum_{v} \bar{\chi}(x+v) t^{a} \chi(x+v)$,

where the sum over $v$ is over the 32 possible lattice displacements of length " $\sqrt{3}$ ". The third group contains 8 operators:

$$
\begin{array}{r}
\mathcal{F}_{i}=\sum_{x, a} \sum_{\mu} \mathcal{C}_{\mu}^{a}(x) \frac{1}{256} \sum_{c} w(c) \eta_{5}(c) \\
P_{\mu}^{(i)}(c) \mathcal{C}_{\mu}^{a}(x+c) \\
i=3, \cdots, 10 .
\end{array}
$$

where the sum over $c$ is a sum over the 81 displacements with coordinates $c_{\mu}=-1,0,1$. The weight is:

$w(c)=\prod_{\mu=1}^{4}\left(2-\left|c_{\mu}\right|\right)$.

The fermion bilinear $\mathcal{C}_{\mu}^{a}(x)$ is given by:

$\mathcal{C}_{\mu}^{a}(x)=\bar{\chi}(x) t^{a} \sum_{v \perp \hat{\mu}} \chi(x+v)$,

where the sum is over the 8 possible lattice displacements of length " $\sqrt{3}$ " which are perpendicular to $\hat{\mu}$ direction. The last group also contains 8 operators:

$$
\begin{array}{r}
\mathcal{F}_{i}=\sum_{x, a} \sum_{\mu} \mathcal{B}_{\mu}^{a}(x) \frac{1}{256} \sum_{c} w(c) \\
P_{\mu}^{(i)}(c) \mathcal{B}_{\mu}^{a}(x+c), \\
i=11, \cdots, 18,
\end{array}
$$

with the fermion bilinear

$\mathcal{B}_{\mu}^{a}(x)=\frac{1}{2}\left[\bar{\chi}(x) t^{a} \chi(x+\hat{\mu})+\bar{\chi}(x) t^{a} \chi(x-\hat{\mu})\right]$.

\subsection{Tree-level values}

At the tree-level, we got:

$$
\begin{array}{rlrl}
c_{0} & =\frac{5}{3}, & c_{1}=-\frac{1}{12}, & b_{1}=-\frac{1}{6}, \\
b_{12}^{\prime}=\frac{g_{0}^{2}}{8}, & b_{13}^{\prime}=\frac{g_{0}^{2}}{24}, & b_{14}^{\prime}=\frac{g_{0}^{2}}{16} .
\end{array}
$$

All other coefficients vanish at the tree-level.

For staggered fermions, the high-momentum gluon exchange gives rise to flavor changing fourfermion terms. However, these terms belong to a restricted class of operators which can be expressed as a product of two fermion bilinears, with each such bilinear composed of fields $\bar{\chi}(x)$ and $\chi\left(x^{\prime}\right)$ with a distance between $x$ and $x^{\prime}$ of precisely ONE link. As we will point out in the next section, all the coefficients of the 8 such terms in Eq. (17) can be changed by a transformation of the field variables and a choice of fields can be found for which these coefficients are zero. Thus, the 8 terms in Eq. (17), including the three treelevel terms quoted above, actually do not appear in the on-shell improved action.

\footnotetext{
${ }^{1}$ We thank G. P. Lepage for pointing out the existence of
} such tree-level contributions. 


\subsection{On-shell improved action}

Given one improved action, we can obtain another one by a transformation of the fields. However, all these actions are equivalent because all such actions will give the same value for a specific on-shell quantity. Thus, we can choose to minimize the number of operators occurring in the on-shell improved action by an appropriate definition of the field variables.

By redefining both gauge and fermion field variables, we find the following operators are redundant: $\mathcal{O}_{2}, \mathcal{O}_{3}, \mathcal{O}_{5}, \mathcal{O}_{6}, \mathcal{F}_{11} \ldots \mathcal{F}_{18}$. So, we can always find a spectrum conserving transformation of gauge and fermion fields such that the following coefficients can be set to be zero:

$b_{2}=b_{3}=b_{5}=b_{6}=0$

$$
b_{11}^{\prime}=\cdots=b_{18}^{\prime}=0 .
$$

Can we transform away more four-fermion operators? The answer is "no". Because in the staggered fermion action, $\bar{\chi}$ and $\chi$ are separated by only one link. After the field transformation, the $\bar{\chi}$ and $\chi$ in the fermion bilinears in the resulting four-fermion operators are separated by only one link also. Hence, only the four-fermion operators in Eq. (17) can be redundant.

Under the field transformation, operators $\mathcal{L}_{3}$ can give a contribution to $\mathcal{O}_{4}$, so we can either set $c_{3}=0$ or $b_{4}=0$. Thus we have two equivalent improved actions:

$$
\begin{gathered}
S^{I}=c_{0} \mathcal{L}_{0}+c_{1} \mathcal{L}_{1}+c_{2} \mathcal{L}_{2} \\
+a^{2}\left[b_{1} \mathcal{O}_{1}+b_{4} \mathcal{O}_{4}+b_{7} \mathcal{O}_{7}+\sum_{i=1}^{10} b_{i}^{\prime} \mathcal{F}_{i}\right]
\end{gathered}
$$

or

$$
\begin{aligned}
S^{I I} & =c_{0} \mathcal{L}_{0}+c_{1} \mathcal{L}_{1}+c_{2} \mathcal{L}_{2}+c_{3} \mathcal{L}_{3} \\
& +a^{2}\left[b_{1} \mathcal{O}_{1}+b_{7} \mathcal{O}_{7}+\sum_{i=1}^{10} b_{i}^{\prime} \mathcal{F}_{i}\right]
\end{aligned}
$$

In either case, the total number of dimension- 6 operators is 15 .

\section{CONCLUSIONS}

The on-shell improved action for lattice QCD with staggered fermions contains 15 dimension six operators. Ten of these are four-fermion operators, which are absent at the tree-level, and hence of the order of $O\left(g_{0}^{4} a^{2}\right)$ at most. The other 5 are fermion bilinears and gauge operators and only two of them are nonzero at tree-level. The numerical results from the MILC and Bielefeld groups presented during this conference are consistent with our analysis.

I thank Prof. Norman H. Christ for the extensive discussions during every stage of this work. This research was supported by the US Department of Energy under grant DE-FG02-92 ER40699.

\section{REFERENCES}

1. K. Symanzik, Nucl. Phys. B226(1983)187, 205.

2. M. Lüscher and P. Weisz, Commun. Math. Phys. 97, 59 (1985).

3. B. Sheikholeslami and R. Wohlert, Nucl. Phys. B259,572(1985).

4. S. Naik, Nucl. Phys. B316, 238(1989).

5. O. Napoly, Phys. Lett. 132B, 145(1983).

6. S. Sharpe, Nucl. Phys. B(Proc. Suppl.) 34, 403(1994),

Yubing Luo, Phys. Rev. D 55, 353(1997).

7. Yubing Luo, Phys. Rev. D, to appear. hep/lat-9702013.

8. T. Blum et al, Phys. Rev. D 55, 1133(1997). 